\title{
Macrófitas e seu potencial fitorremediativo em estações de tratamento de esgoto: uma revisão bibliográfica
}

\author{
Macrophytes and their phytoremediative potential in sewage treatment plants: a literature review \\ Macrófitos y su potencial fitorremediador en plantas de tratamiento de aguas residuales: una \\ revisión de la literatura
}

Recebido: 07/01/2022 | Revisado: 11/01/2022 | Aceito: 18/01/2022 | Publicado: 21/01/2022

\author{
Micaella Assad Ferreira \\ ORCID: https://orcid.org/0000-0002-8048-8514 \\ Universidade da Amazônia, Brasil \\ E-mail: micaellaassad24315@gmail.com \\ Thais Costa Lopes \\ ORCID: https://orcid.org/0000-0002-6832-252X \\ Universidade da Amazônia, Brasil \\ E-mail: lopeste623@gmail.com \\ Dryelle de Nazaré Oliveira do Nascimento \\ ORCID: https://orcid.org/0000-0002-9338-8125 \\ Universidade Federal do Pará, Brasil \\ E-mail: dryelleoliveira@yahoo.com.br \\ Ana Paula Simões Castro \\ ORCID: https://orcid.org/0000-0001-5388-6755 \\ Universidade da Amazônia, Brasil \\ E-mail: biopaulacastro@gmail.com
}

\begin{abstract}
Resumo
$\mathrm{Na}$ atualidade o município de Belém conta com apenas 0,78\% dos esgotos tratados, ressaltando que a capital sempre apresentou escassez no sistema de saneamento básico. Diante disto, podemos perceber as dificuldades existentes nesse setor, que é de extrema importância pra qualidade de vida da sociedade e para o meio ambiente como um todo. Logo, o presente trabalho tem como objetivo estudar o potencial fitorremediativo de macrófitas, analisando a possibilidade de inserção em ETE's. A pesquisa será desenvolvida através de uma revisão de literatura para consultar quais plantas são mais viáveis para inserção em estações de tratamento de esgoto, analisando vários quesitos relevantes para tal. Por meio desta pesquisa pretende-se obter resultados positivos que possam ser aplicados futuramente em ETE's e tragam benefícios para o meio ambiente e para a sociedade no geral, além de trazer informações e esclarecimentos sobre a técnica de fitorremediação, que está sendo cada vez mais utilizada no Brasil.
\end{abstract}

Palavras-chave: Fitorremediação; Saneamento básico; Meio ambiente.

\begin{abstract}
Currently, the municipality of Belém has only $0.78 \%$ of treated sewage, noting that the capital has always had a shortage in the basic sanitation system. Given this, we can see the difficulties that exist in this sector, which is extremely important for the quality of life of society and for the environment as a whole. Therefore, this work aims to study the phytoremediative potential of macrophytes, analyzing the possibility of insertion in ETE's. The research will be developed through a literature review to consult which plants are more viable for insertion in sewage treatment plants, analyzing several relevant questions for such. Through this research, it is intended to obtain positive results that can be applied in the future in ETE's and bring benefits to the environment and society in general, in addition to providing information and clarification on the phytoremediation technique, which is being increasingly used in Brazil. Keywords: Phytoremediation; Basic sanitation; Environment.
\end{abstract}

\section{Resumen}

Actualmente, el municipio de Belém tiene solo el 0,78\% de las aguas residuales tratadas, destacando que la capital siempre ha tenido escasez en el sistema de saneamiento básico. Ante esto, podemos ver las dificultades que existen en este sector, de suma importancia para la calidad de vida de la sociedad y para el medio ambiente en su conjunto. Por tanto, este trabajo tiene como objetivo estudiar el potencial fitorremediador de las macrófitas, analizando la posibilidad de inserción en ETE's. La investigación se desarrollará a través de una revisión de la literatura para consultar qué plantas son más viables para su inserción en plantas de tratamiento de aguas residuales, analizando varias cuestiones relevantes para las mismas. Por meio desta pesquisa pretende-se obter resultados positivos que possam ser aplicados futuramente em ETE's e tragam benefícios para o meio ambiente e para a sociedade no geral, 
além de trazer informações e esclarecimentos sobre a técnica de fitorremediação, que está sendo cada vez mais utilizada en Brasil.

Palabras clave: Fitorremediación; Saneamiento básico; Medio ambiente.

\section{Introdução}

Atualmente os serviços de saneamento básico da cidade de Belém do Pará são realizados pela Companhia de Saneamento do Pará (COSANPA) e a Secretaria Municipal de Saneamento (SESAN). Porém, apesar de existente, o tratamento de esgoto em Belém ainda é insuficiente e não alcança a toda a população do município. De acordo com o Ranking do Saneamento do Instituto Trata Brasil (2019), apenas 0,78 \% do esgoto de Belém apresenta tratamento, se encaixando entre os 21 municípios que tratam $20 \%$ ou menos do seu esgoto, um número que reflete as dificuldades que ainda existem no setor, principalmente em relação a questões de infraestrutura antiga e abandonada, falta de investimento do poder público e falta de segurança adequada nos setores.

Apesar de Belém ser uma região com bastante disponibilidade de água potável, a capital sempre apresentou déficit no sistema de saneamento básico, sendo este sistema responsável pela coleta de resíduos urbanos, drenagem urbana, tratamento de água e efluentes domésticos, apresentando maior déficit se tratando de esgotamento sanitário, onde o percentual de tratamento devidamente realizado é extremamente baixo, o que demonstra uma insuficiência do serviço prestado e carência do sistema (Souza et al., 2018), em um setor de vital importância para a qualidade de vida da sociedade e para o meio ambiente como um todo.

$\mathrm{Na}$ RMB o tratamento de esgoto sanitário é realizado pela COSANPA em Estações de Tratamento de Esgoto especializadas (ETE's), que têm como objetivo através de todas as suas etapas preliminares, primária, secundária e terciária, estabilizarem a carga orgânica presente no esgoto assim tornando possível o retorno deste efluente ao corpo d'água sem maiores danos ao mesmo (Pereira; Silva, 2018) a fim de se adequarem as legislações ambientais vigentes. Porém, como já dito anteriormente, este sistema encontra-se defasado, sendo necessário adotar medidas para melhorar os indicadores de saneamento básico no Estado, pois os efluentes líquidos necessitam receber o devido tratamento, uma vez que são recursos que além de tratados, devem ser reaproveitados, pois os constituintes que se encontram nos esgotos são altamente poluentes se retornados sem tratamento aos corpos d'água (Mackenzie \& Suzan, 2016).

Além disso, há peculiaridades como a extensão territorial e diversidade do Brasil, onde cada região possui diferentes climas, vegetações, recursos naturais, entre outros. Analisando essa diversidade, é possível questionar, por exemplo, se o tratamento realizado na Região Sul do país é realmente o mais adequado ou viável para ser aplicado de forma idêntica no Norte do país. Consequentemente é válido um segundo questionamento: o atual tratamento realizado nas Estações de Tratamento de Esgoto (ETE's) da cidade Belém pode ser modificado, ou melhor, adaptado para a nossa região, com adequações para as especificidades locais e as usando a favor do processo de tratamento sanitário. Uma dessas adaptações seria a inserção de plantas regionais com capacidade fitorremediativas dentro de uma ou mais etapas de tratamento de esgoto ou como etapa adicional.

A fitorremediação é uma técnica que consiste na descontaminação de áreas contaminadas a partir de plantas (Mandalão et al., 2013). Carvalho Neta, et al., (2021), apontou o uso de óleos essenciais com potencial de remediação, especialmente com citotóxicos. O uso de plantas com potencial para remover, degradar ou isolar contaminantes é uma técnica atraente, economicamente viável e de menor custo (Yan et al., 2020). Alguns trabalhos, como o de Souza et al., (2021), constataram que algumas espécies aquáticas, como $S$. polyrhiza e $R$. natans, foram capazes de reduzir concentrações significantes de ferro e manganês.

A técnica tem sido bastante utilizado também para a descontaminação de efluentes e esgoto sanitário principalmente em outros países, onde existem as Wetlands (Pântanos) construídas, que são ecossistemas projetados com plantas aquáticas e 
substratos para simular Wetlands naturais, dentro de uma ou mais etapas do tratamento de esgoto, no intuito de melhorar o tratamento do efluente combinando processos físicos, químicos e biológicos (Poças, 2015). Este tipo de tratamento alternativo já vem sendo estudado e testado aqui no Brasil há certo tempo, citando vantagens e desvantagens do método em que as Wetlands são sistemas aptos que podem ser aplicados para o polimento no tratamento de águas residuárias e que vem ganhando cada vez mais espaço dentro do sistema de tratamento (Poças, 2015).

Portanto, a aplicação de algumas espécies de plantas para despoluir ambientes contaminados não é uma prática tão recente, havendo registros onde há séculos atrás já faziam o uso de determinadas espécies para tratamento de águas residuais (Hartman, 1975 apud Lazart, 2000).

Uma espécie acessível que pode ser encontrada facilmente em todo o Brasil e pode ser utilizada no tratamento de efluentes é a espécies Sansevieriatrifasciata (Liliaceae), popularmente conhecida como espada-de-são-jorge. Sansevieriatrifasciata é uma herbácea de origem africana e consegue ser mantida exposta ao sol ou a meia sombra, mostra uma boa resistência a solos secos e ao calor tropical, como também ao frio, pela facilidade de adaptação em diferentes ambientes. Mostra uma variedade grande de folhas com margens creme-amareladas, com manchas transversais, acinzentadas. É longa em forma de espiga, com pequenas flores brancas (Lorenzi; Souza, 2001).

Diante do exposto, nota-se a necessidade de associar as condições de precariedade apresentadas pelo sistema de esgotamento sanitário regional, com a análise do potencial fitorremediativo de macrófitas identificando sua futura possibilidade de implantação em ETE's, sendo um ponto de partida no intuito de fornecer alternativas complementares de apoio e trazer melhorias para o sistema de saneamento básico da cidade, cuidando do meio ambiente e melhorando a qualidade de vida da população.

\section{Metodologia}

Trata-se de um artigo de revisão bibliográfica narrativa, onde foi pesquisado sobre macrófitas e seu potencial fitorremediativo para o possível uso em estações de tratamento de esgoto na região. Como suporte para este artigo foi utilizada a metodologia descrita por Pereira et al., (2018).

Nesta pesquisa foram analisados livros, documentos governamentais, teses, dissertações e artigos científicos pesquisados em base de dados como o Google Acadêmico e Scientific Eletronic Library Online- Scielo. Foram incluídos artigos e textos publicados de 2000 a 2021 (Figura 1).

Figura 1. Número de trabalhos encontrados e utilizadas no presente trabalho, por ano em que foram publicados.

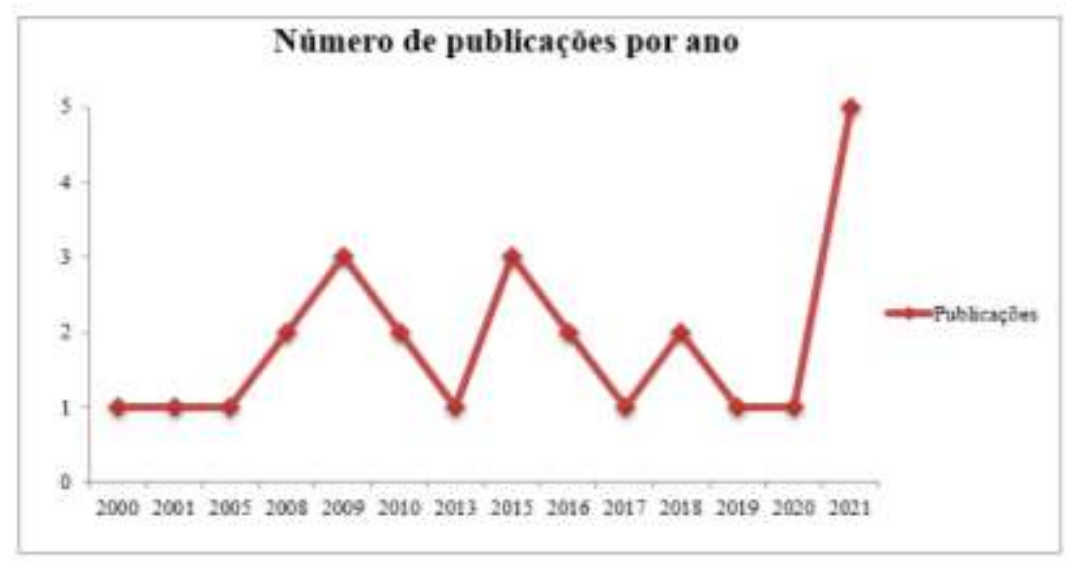

Fonte: Autores. 
No início os termos utilizados para o ponto de partida da pesquisa foram: "tratamento de esgoto", "saneamento básico na região Norte", "fitorremediação", "macrófitas", "macrófitas e seu potencial fitorremediativo", "macrófitas em estações de tratamento de esgoto". A partir disso, foram selecionados temas mais específicos discorrendo sobre as espécies de macrófitas que podem ser utilizadas para a realização da fitorremediação (Figura 2) em estações de tratamento de esgoto como: "Typhaspp.", "Vetiveriazizanioides L. Nash", "Eichhorniacrassipes", "Hedychiumcoronarium", "Heliconiapsittacorum", "Cyperusalternifolius".

Figura 2. Fluxograma exemplificando a linha de pesquisas feita nos bancos de busca.

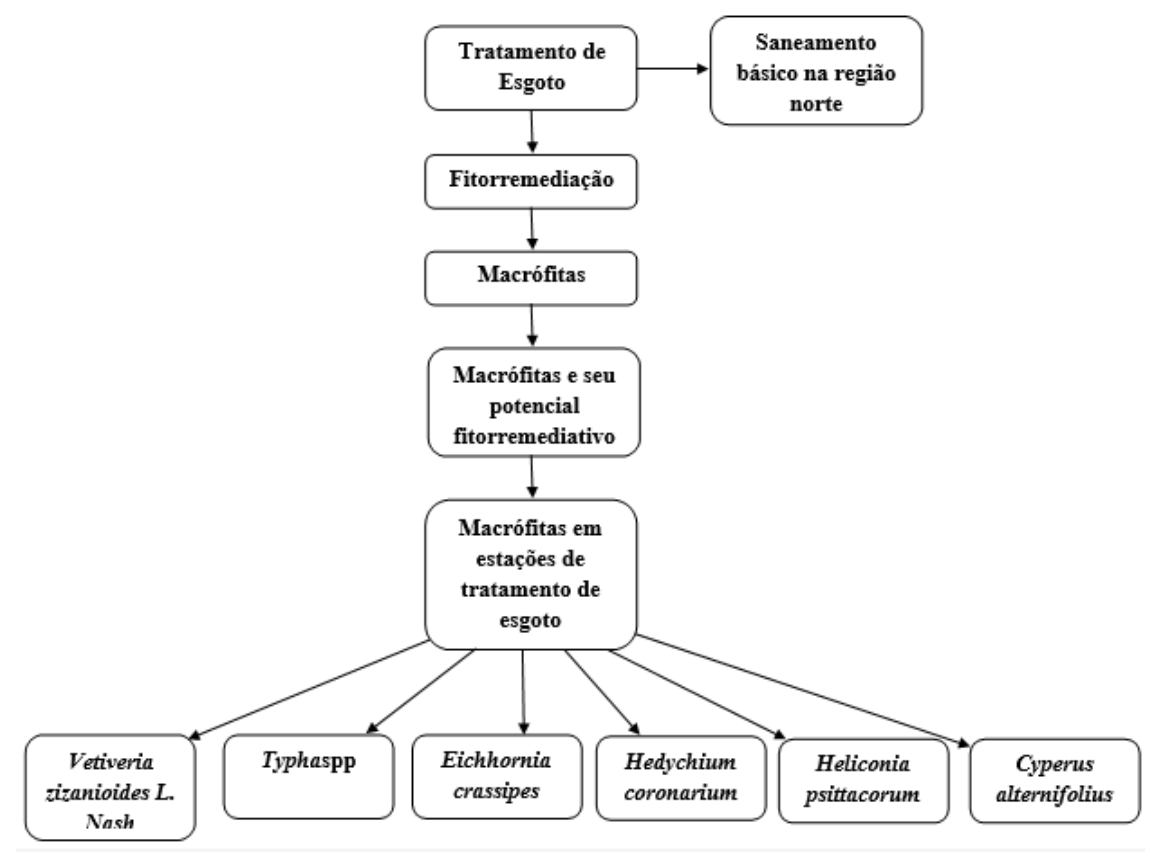

Fonte: Autores.

Foram excluídos desta pesquisa artigos indisponíveis na íntegra ou que tivessem teor político. Os resultados foram expressos de maneira descritiva (Figura 3).

Figura 3. Tabela ilustrativa dos termos pesquisados e os principais artigos encontrados no presente trabalho.

\begin{tabular}{|c|c|}
\hline Tepicen: & Anteres \\
\hline Tratumeno dr Evequta & 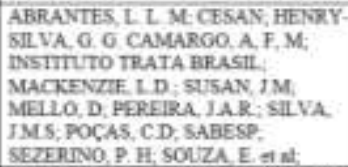 \\
\hline Fitoermaediacllo & 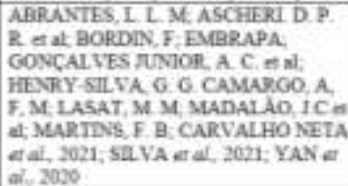 \\
\hline Mandófites & 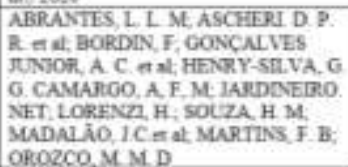 \\
\hline Wellont & 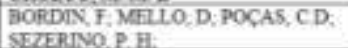 \\
\hline
\end{tabular}

Fonte: Autores. 


\section{Resultados e Discussão}

\subsection{Macrófitas}

Segundo Sezerino et al., (2015, p. 155), não existe uma regra para a implementação das macrófitas dentro dos sistemas de Wetlands construídos (SWC), porém é importante que sejam retiradas as plantas que não vingaram durante o transplante do ambiental natural para o artificial.

De acordo com publicação da Iwa (2000 apud Mello, 2016), dentro de um Wetland as macrófitas são a principal fonte de tratamento por meio da assimilação de poluentes.

Segundo Abrantes (2009), as plantas devem proporcionar uma relação mutualística com bactérias aeróbias por meios de suas raízes para que elas se fixem e obtenham oxigênio, retirar água e absorver nutrientes.

As plantas nos Wetlands construídos necessitam de nutrientes para seu crescimento e nutrição, assim os nutrientes presentes nos esgotos são assimilados por elas e se transformam em biomassa das macrófitas que podem ser aproveitadas em outros meios após serem colhidas. É de extrema importância que as macrófitas sejam colhidas de forma periódica, pois os nutrientes que elas absorveram podem retornar ao ambiente aquático com a decomposição de seus tecidos (Abrantes, 2009).

\subsection{Fitorremediação}

Embora o termo fitorremediação seja mais recente, essa técnica já era utilizada na antiguidade, por exemplo, pelos egípcios para auxiliar na despoluição de esgotos urbanos que desembocavam nas águas do Rio Nilo, uma vez que este processo consiste na capacidade de absorção das raízes das plantas não apenas de água e nutrientes que colaboram para o seu crescimento, mas também de poluentes que fazem com que esses vegetais se tornem filtros biológicos, onde os principais exemplos são metais pesados e o petróleo, valendo ressaltar a importância de identificar corretamente as substâncias que estão poluindo o ambiente para que seja cultivada a espécie correta, de acordo com a Embrapa (2010).

Porém, como dito no tópico anterior, para que a fitorremediação seja efetiva é necessário que exista um controle das plantas que fazem parte desde processo, pois caso elas não sejam retiradas de forma periódica do ambiente no qual foram colocadas, os nutrientes e poluentes que foram absorvidos por elas irão retornar ao meio por causa da decomposição do vegetal.

\subsection{Tratamento de Esgoto}

O tratamento de esgoto é realizado para que ocorra a remoção de poluentes despejados de residências e indústrias. Segundo descrições de companhias de saneamento, como a Sabesp e Cesan, podemos observar que a forma de tratamento de esgoto no Brasil segue o mesmo padrão, iniciando-se pelo recebimento dos efluentes na Estação de Tratamento de Esgoto (ETE) para que seja realizada a separação de resíduos sólidos que podem ser facilmente retirados por um sistema de gradeamento. Em seguida é feita a aeração do esgoto, motivando o crescimento de colônias de bactérias aeróbias que irão degradar a matéria orgânica e formando o lodo. Após esse processo, inicia-se a separação da água e do lodo por meio da decantação, a matéria que se precipita durante a decantação recebe o nome de biosólido e pode ser destinado para a agricultura. Por fim, a água passa por um processo de desinfecção com raios ultravioleta para a remoção dos microrganismos, assim a água é destinada para a reutilização, principalmente, na área da construção civil, evitando a utilização de água potável nessas atividades.

Contudo, ainda existem diversos locais que não possuem eficiência durante o processo de tratamento de esgoto, de acordo dados do Ranking do Saneamento Instituto Trata Brasil (2019) podemos perceber que diversos municípios brasileiros necessitam de mais investimentos ou mudanças nos processos de tratamento de esgoto, valendo ressaltar a presença de três municípios paraense que são: Belém, Santarém e Ananindeua. 


\subsection{Fitorremediação em águas residuárias}

Quando falamos sobre a utilização de plantas como forma de auxiliar o tratamento de esgoto nas cidades, é indispensável citarmos os sistemas de Wetlands construídos que agem como filtros biológicos durante o processo de tratamento dos efluentes. É de extrema importância ressaltar que esse sistema não vem para substituir o processo que ocorre nas ETE's, mas sim complementar e melhorar as fases que ocorrem à redução de matéria orgânica e de poluentes.

Os Wetlands possuem diversas vantagens, como: o baixo custo de implantação e manutenção, além do Brasil possuir condições climáticas e ambientais excelentes para os Wetlands. Além das vantagens econômicas, é extremamente importante ressaltar os benefícios durante o processo de tratamento de efluentes que, segundo Poças (2015), podemos listar: desnitrificação motivada pela remoção do nitrato; íons de amônio e metais ficam retidos na superfície de minerais encontrados na porção argilosa do solo; o mesmo ocorre com outros íons metálicos, pesticidas e compostos com fósforo, porém com a ação da matéria orgânica; decomposição da matéria orgânica biodegradável, por ação aeróbia e anaeróbia; alguns microrganismos auxiliam na remoção de patógenos, retirada de metais pesados e toxinas por meio das macrófitas.

Com base no que foi exposto, fica evidente a praticidade de se adotar sistemas de Wetlands construídos ao longo de um processo padrão de ETE's, pois significa uma alternativa economicamente viável que irá melhorar a qualidade final dos efluentes, além das plantas utilizadas durante o processo poderem ser utilizadas como alimento na agropecuária ou até mesmo como decoração de alguns espaços.

\subsection{Eichhorniacrassipes}

A Eichhorniacrassipes é uma planta nativa da região Amazônica e foi difundida pelo mundo devido à beleza ornamental das suas flores (Szymanski, 2011 apud Mello, 2016). E por mais que seja típica da região Amazônica, essa macrófita pode ser encontrada ao longo de todo o território brasileiro.

Segundo Martins, et al. (2005) e Gonçalves Júnior et al. (2008), um dos benefícios da utilização dessa planta é o seu rápido crescimento em ambientes aquáticos com grande disponibilidade de nutrientes que serão utilizados em seu metabolismo, além de agirem na fitorremediação de metais pesados e outros contaminantes que elas são capazes de absorver. Gonçalves Júnior et al. (2008) utiliza essas características como justificativa para a maior utilização da Eichhornia crassipes no tratamento de efluentes. Sipaúba-Tavares et al. (2001, apud Mello, 2016) e Henry-Silva e Camargo (2008) utilizaram a Eichhornia crassipes no tratamento de diferentes tipos de efluentes, provando que a utilização desta espécie está em constante avanço no tratamento de efluentes.

\subsection{Cyperus alternifolius}

Nativa de Madagascar possui facilidade para se adaptar em diversos ambientes uma vez que suporta climas mais quentes e também mais frios, não tem exigência de $\mathrm{pH}$ e cresce aproximadamente até 1,5 metro de altura de forma muito rápida, além da possuir versatilidade para a sua reprodução. Contudo, a Cyperus alternifolius não se comporta bem com podas frequentes, não conseguindo retomar seu tamanho original após sucessivas podas (Jardineiro.net, 2015).

Durante um experimento utilizando a Cyperus alternifolius em conjunto com outras macrófitas em um Wetland construído na região de Campinas-SP, foi observado a eficiência da extração de nutrientes em diferentes situações, assim como o ganho de biomassa das plantas analisadas. A Cyperus alternifolius se mostrou uma excelente opção principalmente antes de ser podada durante o experimento, apresentando altas taxas de extração de fósforo e nitrogênio amoniacal (Kleteckee, 2011 apud Poças, 2015, p. 53-54). 


\subsection{Hedychium coronarium}

Esta macrófita é nativa da Ásia tropical e, segundo a definição de Ascheri (2009), necessita de solos ricos em matéria orgânica e permanentemente molhados, porém não se trata de uma macrófita que se localiza abaixo da água, é uma macrófita emersa. Pode ser cultivada em locais com muito sol, mas apresenta potencial invasivo se não for controlada.

De acordo com os dados obtidos por Kleteckee (2011, apud Poças, 2015, p. 53-54) durante a mesma pesquisa citada quando tratamos sobre a Cyperusalternifolius, podemos perceber que a Hedychiumcoronarium apresenta uma eficiência bem semelhante a da macrófita citada anteriormente, porém apresenta dados melhores após a poda, principalmente na remoção de nitrogênio amoniacal e na Demanda Química de Oxigênio (DQO).

\subsection{Heliconia psittacorum}

Mais uma planta nativa do Brasil, aprecia calor e umidade típicos de um clima tropical, mas tolera climas mais frios como o subtropical. Costuma forma grandes touceiras (moitas) que alcançam até 2 metros de altura, possui inflorescências curtas e duráveis durante o período do verão. Pode ser cultivada exposta ao sol ou em meia sombra, com solo enriquecido com matéria orgânica e mantido úmido.

Dentre as duas macrófitas anteriores que foram citadas, a Heliconiapsittacorum foi a que obteve melhores resultados gerais durante o experimento de Kleteckee (2011 apud Poças, 2015, p. 53-54), destacando-se na manutenção de valores da remoção de DQO antes e depois da poda, menor variação na retenção de fósforo antes e depois da poda, e por acumular maior quantidade (em gramas) de nitrogênio após a poda para a extração. Sendo esse último dado bem relevante, uma vez que a biomassa acumulada pelas macrófitas costuma ser reutilizada na agricultura e, possuindo uma grande reserva de nitrogênio, essa planta pode ser uma alternativa para a suplementação de nitrogênio no solo que será convertido em nitratos e nitritos, por meio do ciclo do nitrogênio, para que as outras plantas possam absorver.

\subsection{Outras macrófitas}

Além das plantas já citadas, existem diversas outras macrófitas que podem ser utilizadas quando o objetivo é potencializar o processo de tratamento dos efluentes. Por exemplo, temos a Vetiveria zizanoídes L. Nash que pode ser chamada de Capim Vetiver, que se trata de uma gramínea típica da Índia e utilizada há mais de 3 mil anos nessa região por ser uma planta extremamente resistente, podendo suportar secas, uma vez que suas raízes são profundas, resiste a uma faixa de $\mathrm{pH}$ que varia de 3,0 até 10,5, tolera altos níveis de metais e pesticidas, além de ser muito eficiente na retirada de nitrogênio e fósforo em ambientes poluídos (Orozco, 2009).

A taboa é outra macrófita que é muito utilizada para o tratamento da água, possui diversas espécies (Typhaspp.) que desempenham esta função. É considerada uma planta daninha aquática e, por ter diversas espécies, podemos encontrá-la distribuída em vários continentes. Sua importância está na sua grande capacidade de absorção de metais pesados (Bordim, 2010).

\section{Considerações Finais}

A utilização de macrófitas através de Wetlands dentro de estações de tratamento de esgoto como um tratamento adicional ou complementar, tem se mostrado uma alternativa economicamente viável e que melhoraria muito a qualidade do efluente que retornará ao meio ambiente. Esta revisão destaca as principais vantagens da aplicação destas macrófitas dentro de sistemas de Wetlans e sua eficiência associadas ao tratamento de esgoto, explanando prós e contras em sua utilização e buscando trazer alternativas viáveis para a melhoria do tratamento de esgoto na região norte que como já dito anteriormente, infelizmente apresenta baixo índice de tratamento. 
Assim sendo, conclui-se que é viável a implantação de macrófitas através de sistemas de Wetlands construídas em estações de tratamento de esgoto da região. No entanto, para uma correta e segura inserção destas é necessário e recomendado que haja a realização de mais estudos na área, com a aplicação de pesquisas em campo e a realização de análises com as macrófitas e suas respectivas capacidades de fitorremediação de acordo com o poluente ao qual serão expostas, para assim haver a possibilidade de uma real implantação em ETE's.

\section{Referências}

Abrantes, L. L. M. (2009) Tratamento de esgoto sanitário em sistemas alagados construídos utilizando Typha angustifólia e Phragmites australis. Dissertação (Mestrado) - Universidade Federal de Goiânia, Programa de Pós-Graduação Stricto Sensu em Engenharia do Meio Ambiente, Goiânia. http://repositorio.bc.ufg.br/tede/bitstream/tde/624/1/Dissertacao\%20Lorena\%20L\%20M\%20Abrantes.pdf

Ascheri. D. P. R. et al. (2009). Propriedades termodinâmicas de adsorção de água do amido de rizomas do lírio-do-brejo (Hedychium coronarium). Ciênc. Tecnol. Aliment, 29(2): 454-462. https://www.scielo.br/j/cta/a/znWsJsXdmzmzfVPJshnD6cr/?lang=pt\&format=pdf.

Bordin, F. (2010) Estudo da remoção de matéria orgânica carbonácea, nitrogênio amoniacal e fósforo em lixiviado pré-tratado de aterro sanitário empregando typha em Wetland. 124 f. Dissertação (Mestrado) - Universidade do Rio do Vale dos Sinos, Programa de Pós-Graduação em Engenharia Civil, São Leopoldo. http://www.repositorio.jesuita.org.br/bitstream/handle/UNISINOS/4509/FabianeBordin.pdf?sequence=1\&isAllowed=y.

Carvalho Neta, R. N. F et al., (2021). Potential uses of essential oils in environmental remediation: A review. Research, Society and Development, 10(7), e3210716146, 10.33448/rsd-v10i7.16146. https://rsdjournal.org/index.php/rsd/article/view/16146.

Companhia Espírito Santense de Saneamento (2017). Confira como é feito o processo de tratamento do esgoto. https://www.cesan.com.br/noticias/confiracomo-e-feito-o-processo-de-tratamento-do-esgoto/

EMBRAPA (2012). Prosa Rural - Fitorremediação: o uso de plantas para descontaminação ambiental. https://www.embrapa.br/busca-de-noticias//noticia/2419877/prosa-rural---fitorremediacao-o-uso-de-plantas-para-descontaminacao-ambiental.

Gonçalves Junior, A. C. et al. (2008). Remoção de metais pesados tóxicos cádmio, chumbo e cromo em biofertilizante suíno utilizando macrófita aquática (Eichornia crassipes) como bioindicador. Acta $\quad$ Sci. https://www.researchgate.net/publication/26598009_Remocao_de_metais_pesados_toxicos_cadmio_chumbo_e_cromo_em_biofertilizante_suino_utilizando_ macrofita_aquatica_Eichornia_crassipes_como_bioindicador_Removal_of_toxic_heavy_metals_cadmium_lead_and_chro.

Henry-Silva, G. G. \& Camargo, A, F, M. (2008). Tratamento de efluentes de carcinicultura por macrófitas aquáticas flutuantes. R. Bras. Zootec, 37(2), 181188. https://www.scielo.br/j/rbz/a/Vwgff8G8nW6DCQhCN5h385H/?format=pdf\&lang=pt.

Instituto Trata Brasil (2017). O $\quad$ que é saneamento. http://www.tratabrasil.org.br/saneamento/o-que-esaneamento\#: :text=Saneamento\%20\%C3\%A9\%20o\%20conjunto\%20de,e\%20facilitar\%20a\%20atividade\%20econ\%C3\%B4mica.

Instituto Trata Brasil (2019). Ranking do saneamento instituto trata Brasil. São Paulo. http://tratabrasil.org.br/images/estudos/itb/ranking2019/Relat\%C3\%B3rio_-_Ranking_Trata_Brasil_2019_v11_NOVO_1.pdf.

Jardineiro.net (2015). Sombrinha-chinesa - Cyperus alternifolius. https://www.jardineiro.net/plantas/sombrinha-chinesa-cyperus-alternifolius.html.

Lasat, M. M. (2000). The Use of Plants for the Removal of Toxic Metals from Contaminated Soils. American Association for the Advancement of Science Environmental Science and Engineering Fellow. https://www-semanticscholar-org.translate.goog/paper/The-Use-of-Plants-for-the-Removal-of-Toxic-MetalsLasat-Lasat/b2c671c4fd35812aa98370afd8009d79b94af434?_x_tr_sl=en\&_x_tr_tl=pt\&_x_tr_hl=pt-BR\&_x_tr_pto=sc.

Lorenzi, H. \& Souza, H. M. (2001). Plantas Ornamentais no Brasil: arbustivas, herbáceas e trepadeiras. $3^{a}$ ed. Nova Odessa: Instituto Plantarum.

Mackenzie, L.D. \& Susan, J.M. (2016). Princípios de Engenharia Ambiental. (3a ed.), AMGH.

Madalão, J.C.et al. (2013). Susceptibilidade de espécies de plantas com potencial de fitorremediação do herbicida sulfentrazonel. Rev. Ceres, 60(1), 111-121. https://www.scielo.br/pdf/rceres/v60n1/16.pdf.

Martins, F. B. et al. (2005). Zoneamento ambiental da sub-bacia hidrográfica do Arroio cadena, Santa Maria (RS) - (estudo de caso). Cerne, Lavras, v. 11, n. 3, p. 315-322. http://repositorio.ufla.br/jspui/bitstream/1/14540/1/ARTIGO_Zoneamento\%20ambiental\%20da\%20subbacia\%20hidrogr\%c3\%a1fica\%20do\%20Arroio\%20Cadena\%2c\%20Santa\%20Maria\%20\%28RS\%29\%20\%28 estudo\%20de\%20caso $\% 29$.pdf.

Mello, D. (2016). Avaliação do uso de sistemas de wetlands construídas no tratamento de esgotos sanitários. 161 f. Dissertação (Mestrado) - Universidade Tecnológica Federal do Paraná. Programa de Pós-Graduação em Ciência e Tecnologia Ambiental, Curitiba. https://repositorio.utfpr.edu.br/jspui/bitstream/1/1840/1/CT_PPGCTA_M_Mello\%2C\%20D\%C3\%A9bora\%20de_2016.pdf.

Orozco, M. M. D. (2009). Caracterização da gramínea vetiveria zizanioides para aplicação na recuperação de áreas degradadas por erosão. 2009 . 81 f. Dissertação (Mestrado) - Universidade Federal de Minas Gerais, Escola de Engenharia, Programa De Pós-Graduação em Saneamento, Meio Ambiente e Recursos Hídricos, Belo Horizonte. Disponível em: https://repositorio.ufmg.br/bitstream/1843/FRPC-82SGNK/1/760m.pdf.

Pereira, J.A.R. \& Silva, J.M.S. (2018). Rede coletora de Esgoto Sanitário: Projeto, Construção e Operação. (3a ed.), Belém.

Poças, C. D. (2015). Utilização da tecnologia de Wetlands para tratamento terciário: Controle de nutrientes. 93 f. Dissertação (Mestrado) - Faculdade de Saúde Pública da Universidade de São Paulo, São Paulo. https://www.teses.usp.br/teses/disponiveis/6/6139/tde-23112015-122556/pt-br.php. 
Research, Society and Development, v. 11, n. 2, e13711225457, 2022

(CC BY 4.0) | ISSN 2525-3409 | DOI: http://dx.doi.org/10.33448/rsd-v11i2.25457

Companhia de Saneamento Básico do Estado de São Paulo. (2019) Tratamento de esgotos. http://site.sabesp.com.br/site/interna/Default.aspx?secaoId=49.

Sezerino, P. H. et al. (2015). Experiências brasileiras com wetlands construídos aplicados ao tratamento de águas residuárias: parâmetros de projeto para sistemas horizontais. Eng Sanit Ambient, 20(1), 151-158. https://www.scielo.br/j/esa/a/8zwy5WwwLZVxRk5btDHDMkG/?lang=pt\&format=pdf.

Silva, D. M. et al., (2021). Aquatic plants in ecotechnologies: perspectives for phytoremediation of iron and manganese. Research, Society and Development, 10(3), e29510313320, 10.33448/rsd-v10i3.13320. https://rsdjournal.org/index.php/rsd/article/view/13320.

Souza, E. et al. (2018). Panorama situacional do serviço de esgotamento sanitário e sua relação com doenças de veiculação hídrica na região metropolitana de Belém -Pará. RG\&SA, 7(3), 487-503, http://www.portaldeperiodicos.unisul.br/index.php/gestao_ambiental/article/view/6964/4071.

Yan, A. et al., (2020). Phytoremediation: A Promising Approach for Revegetation of Heavy Metal - Polluted Land. Frontiers in Plant Science, 11, 359. 